\title{
Universality and scaling in compressible turbulence
}

\author{
Diego A. Donzi:ª and John Panickacheril John \\ Department of Aerospace Engineering, Texas AËM University, College Station, Texas 77843, USA
}

\begin{abstract}
Turbulent flows, ubiquitous in nature and engineering, comprise fluctuations over a wide range of spatial and temporal scales. While flows with fluctuations in thermodynamic variables are much more common, much less is known about these flows than their incompressible counterparts in which thermodynamics is decoupled from hydrodynamics. A critical element in the study of the latter has been the concept of universal scaling laws which provides fundamental as well as practical information about the spatio-temporal behavior of these complex systems. Part of this success is due to the dependence on a single non-dimensional parameter, that is the Reynolds number. Universality in compressible flows, on the other hand, have proven to be more elusive as no unifying set of parameters were found to yield universal scaling laws. This severely limits our understanding of these flows and the successful development of theoretically sound models. Using results in specific asymptotic limits of the governing equations we show that universal scaling is indeed observed when the set of governing parameters is expanded to include internally generated dilatational scales which are the result of the driving mechanisms that produce the turbulence. The analysis demonstrate why previous scaling laws fail in the general case, and opens up new venues to identify physical processes of interest and create turbulence models needed for simulations of turbulent flows at realistic conditions. We support our results with a new massive database of highly-resolved direct numerical simulations along with all data available in the literature. In search of universal features, we suggest classes which bundle the evolution of flows in the new parameter space. An ultimate asymptotic regime predicted independently by renormalization group theories and statistical mechanics is also assssed with available data.
\end{abstract}

Most flows in nature and engineering are turbulent exhibiting fluctuations in velocity, pressure and virtually all hydrodynamic and thermodynamic variables describing the fluid state. A strong simplification widely used is the assumption of incompressibility, that is, the volume preservation of fluid elements as they deform and rotate in space and time. This condition which implies a solenoidal velocity field and constant density fields, also commonly leads to a decoupling of hydrodynamics and thermodynamics, simplifying the problem greatly. However, this is not the case in the most general condition which includes flows in astrophysical and cosmological contexts, aerodynamics of aircraft and spacecraft, geophysical phenomena such as volcanic eruptions, and reacting turbulent flows, among many others. Due to the extreme complexity of these systems with their wide range of non-linearly interacting spatial and temporal scales, it is only in a statistical sense that these flows are expected to be characterized and understood. Even then, a main obstacle in this endeavor is the apparent difficulty seen in the literature to find universal scaling laws for different flows or for similar flows under diverse conditions. These attempts are commonly based on two non-dimensional parameters, namely, the Taylor Reynolds number $\left(R_{\lambda}\right)$ which represents the relative importance of inertial to viscous effects, and the turbulent Mach number $\left(M_{t}\right)$ which is the ratio of a characteristic velocity to the speed of sound. However, these efforts have remained generally inconclusive and support for different proposals has been limited and often contradictory.

\footnotetext{
* donzis@tamu.edu
}

In most cases, scaling laws are hard to discern and found to depend on the type of driving mechanism that sustains turbulence [1 7], initial conditions [8, 9] or other details of the flow. This is in contrast to the relative success in incompressible turbulence where universal scaling laws have proven insightful and practically useful [10, 11].

As we argue here, universal scaling in compressible turbulence is indeed observed, but only when internally generated scales representing purely compressible motions are the basis for similarity scaling. This represents a departure from previous efforts which focus on externally imposed scales leading to an incomplete set of governing non-dimensional parameters to define the state of turbulence. We show how this state of affairs followed from a few (sometimes implicit) assumptions about the variables that control the system.

In particular we show that to define the state of turbulence, dilatational motions cannot be neglected, as traditionally done. Using first pressure fluctuations, we show that the flow undergoes a transition between two equilibrium states, one dominated by incompressible non-linear dynamics and the other one by compressible or dilatational motions with simple linear dynamics. The scaling on these two limits depends on different set of parameters, one including dilatational motions while the other not. The relative importance of these two equilibria lead naturally to governing parameters that uniquely define the statistical state of fluctuating thermodynamic variables. Classical parameters are also shown to fail to collapse other quantities important to the dynamics of turbulence such the rate at which turbulent kinetic energy is dissipated as well as the rate at which vortical motions are produced which exemplify both the non-linear behavior and conspicuous non-Gaussianity of turbulence. We 
show that universality is also unraveled for these variables by the introduction of dilatational motions into the governing parameters. These, in turn, define statistical regimes in the extended parameter space which help explain discrepancies in the literature and explain when and in what sense universal scaling is expected. The results presented, thus, provide a new perspective for compressible turbulence, and perhaps more general non-linear multiscale systems.

\section{BACKGROUND}

A common paradigm to understand fundamental issues in turbulence is to focus on the intrinsic dynamics of statistically steady isotropic, homogeneous flows to avoid complications from wall effects, spatial nonuniformities and transients. Even in this simplified setup, the extraordinary difficulties associated with rigorous treatments of the non-linear governing equations (that is, the Navier-Stokes equations) have resulted in experiments and numerical simulations being a main driver of progress. In the case of compressible turbulence, experiments under these simplifying assumptions in well controlled conditions are also exceedingly difficult and thus scarce. Therefore, numerical simulations have become a main tool in investigating these flows. Of particular importance are the so-called direct numerical simulations (DNS) where the exact governing equations expressing conservation of mass, momentum and energy are solved such that all temporal and spatial scales are accurately resolved by the numerical scheme [12, [13. Due to the complexity of these equations and the wide range of scales, DNS are computationally extremely expensive and require massive computational power. This is the type of simulations we will use here which, to the authors' knowledge, is among the largest and bestresolved databases of isotropic compressible turbulence in the published literature.

Because of the comparatively larger body of literature in incompressible turbulence and its more limited set of governing parameters and physical processes, a common approach to study compressible flows is to unveil differences from incompressible counterparts by quantifying departures from known scaling laws. This has led to significant advances in the field [14, 15. A practical, yet rigorous, approach to separate aspects associated with incompressible and compressible motions is the Helmholtz decomposition in which the velocity field is split as $\boldsymbol{u}=\boldsymbol{u}_{s}+\boldsymbol{u}_{d}$ where $\boldsymbol{u}_{s}=\nabla \times \boldsymbol{A}(\boldsymbol{A}$ is the vector potential) and $\boldsymbol{u}_{d}=\nabla \phi$ ( $\phi$ is the scalar potential). It is trivial to show that $\boldsymbol{u}_{s}$ is solenoidal (i.e. $\nabla \cdot \boldsymbol{u}_{s}=0$ ) and that $\boldsymbol{u}_{d}$ is irrotational (i.e. $\nabla \times \boldsymbol{u}_{d}=0$ ). Since $\boldsymbol{u}_{d}$ is identically zero in incompressible flows, its existence is ascribed to purely compressibility effects. It is important to note, though, that compressibility can also affect $\boldsymbol{u}_{s}$. While the decomposition of related variables is also possible (e.g. $\sqrt{\rho} \boldsymbol{u}[16$ ]) we have verified that conclusions here are independent of this choice.

Decades of accumulated numerical work, have made some aspects of these flows increasingly clear. A few examples include mixing inhibition due to compressibility 17 19, changes in decay rates due to initial level of dilatation or thermodynamic fluctuations [9, 20, 23], and, when one compares across studies as done here, the failure of traditional parameters (in particular Reynolds and turbulent Mach numbers) to provide universal descriptions these flows.

The purpose of this work is to assess in which sense compressible turbulence can be dubbed universal and under what conditions one can expect universal scaling. In doing so we propose a set of parameters that define new regimes and lead to universal scaling laws that can, indeed, collapse all the data available in the literature. This leads to both the recognition of limitations of current approaches as well as new paths for more general understanding and modeling approaches.

\section{GOVERNING PARAMETERS, SCALING AND SIMILARITY}

In order to explore similarity scaling in compressible flows, we first discuss key fundamental issues involved in its application to their incompressible counterparts. In this case the equations for conservation of mass and momentum, known as the Navier-Stokes equations, are sufficient to describe the phenomenon to great accuracy. In search of similarity, one can use a characteristic velocity $(\mathcal{U})$ and length scale $(\mathcal{L})$ to normalize all variables in the governing equations. This results in a single similarity parameter, the Reynolds number $R=\mathcal{U} \mathcal{L} / \nu$ where $\nu=\mu / \rho$ with $\mu$ being the dynamic viscosity and $\rho$ the (constant) density. Geometrically similar flows are then expected to have identical properties if the Reynolds number is the same. In principle, if scaling laws in $R$ are known and $\mathcal{U}$ and $\mathcal{L}$ are known parameters of the problem at hand (e.g. mean speed and the mesh size in grid turbulence, or the size of an object generating a turbulent wake) then one obtains useful predictive capabilities by simply knowing the geometry and conditions of the flow. Since $\mathcal{L}$ and $\mathcal{U}$ are externally imposed we can call this, external similarity. Dimensional analysis would then imply that an appropriately normalized statistics of interest $Q$, can be represented as $\bar{Q}=f_{i}(R)$ where an overbar denotes normalization and $f_{i}$ is presumably a universal function for incompressible flows.

This approach, however, proves to be limiting when comparisons across different flows is attempted. For example, the normalized mean dissipation rate $\langle\epsilon\rangle \mathcal{L} / \mathcal{U}^{3}$ $(\epsilon$ is the instantaneous dissipation rate per unit mass and angular brackets are suitably defined ensemble averages) is not expected to be the same for a body wake or grid turbulence if $\mathcal{L}$ is the diameter of the body for the former and the grid spacing for the latter though the order of magnitude may be correctly predicted. To 
compare across flow, thus, it is common to use the large-scale Reynolds number $R_{L}=u_{\mathrm{rms}} L / \sqrt{3} \nu$, where $u_{\text {rms }}^{2}=\left\langle|\boldsymbol{u}-\langle\boldsymbol{u}\rangle|^{2}\right\rangle$ (angular brackets are suitably defined ensemble averages) is the root-mean-square velocity and $L$ the integral length scale, or the Taylor Reynolds number $R_{\lambda}=u_{\mathrm{rms}} \lambda / \sqrt{3} \nu$ where $\lambda$ is the Taylor microscale which is an intermediate scale between the largest scales and the smallest dissipative scales. A vast literature on incompressible turbulence has been devoted to elucidating the scaling of different statistical features of turbulence with $R_{\lambda}$, that is:

$$
\bar{Q}=f_{i}\left(R_{\lambda}\right)
$$

Two critical issues are worth noting here. First, there is the implicit assumption that a single velocity scale and a single length scale are enough to completely characterize the flow, at least, in a statistical sense. Second, these flow scales $\left(u_{\mathrm{rms}}, L\right.$ and $\lambda$ ) are computed from the flow itself and their value can only be estimated to within order of magnitude from a priori known geometrical details of the flow setup. One is thus forced to acknowledge the knowledge gap between a priori characteristic velocities and lengths from the original geometry and the resulting internal scales from the flow dynamics subjected to those particular initial or boundary conditions defined by $\mathcal{U}$ and $\mathcal{L}$. When using internally generated characteristic scales, we call this internal or self similarity. While a disadvantage of such an approach is the lack of pure predictive capabilities from a priori known characteristics of the flow, its advantage lies on its ability of unravel universal aspects across geometrically different flows.

A well-known example is the phenomenology of Kolmogorov 24 who suggested internally generated scales (the Kolmogorov scales) as a way to find universality. This universality is indeed observed by the good collapse of multi-point statistics (at least for low orders [10]) when normalized by Kolmogorov scales [25]. One of the most salient examples is the observed collapse of the normalized energy spectrum from a variety of flows at different Reynolds numbers [26].

Most efforts to find universality in compressible turbulence have attempted to use known results from incompressible flows and study departures and differences as compressibility levels increase [14. The degree of compressibilty is commonly measured by the Mach number constructed by a characteristic velocity $\mathcal{U}$, and the speed of acoustic propagation $c$, that is $\mathcal{M} \equiv \mathcal{U} / c$. Clearly for incompressible flows, $\mathcal{M}=0$ since $c \rightarrow \infty$. A wellknown example of this paradigm is the widely studied reduction of the spreading rate of a mixing layer with $\mathcal{M}$ (defined with some average convective velocity) [27]. There is still debate about what the correct definition of $\mathcal{M}$ is, though [14, 28? 30. Given the complexity of turbulence in general and compressible turbulence in particular, a large body of literature has been devoted instead to homogeneous isotropic flows, where no mean velocity exists. This approach avoids the additional complexity of geomertical factors and allows for fundamental under- standing of intrinsic characteristics that emerge from the governing equations. In such flows, which are the focus of this work as well, it is common to characterize the degree of compressibility with the turbulent Mach number $M_{t} \equiv u_{\mathrm{rms}} / c$ where $c$ is the mean speed of sound. This clearly corresponds to self similarity as described above. An implicit assumption here is that the addition of the propagation speed of acoustic waves provides a complete set of governing parameters of the flow. Perhaps a more appropriate interpretation is apparent from the relation $c^{2} \sim\langle T\rangle \sim\langle p\rangle /\langle\rho\rangle$ for a perfect gas. Thus, $c$ completes the specification of the thermodynamic state of the flow (at least in a mean sense) when density is included in the set of governing parameters. Note also that using the mean speed of sound amounts to again seeking internal similarity as $c$, in general, depends on the flow solution which involves temperature fluctuations. Selfsimilar scaling would then imply that there are universal functions $f_{c}$ for different non-dimensional quantities $\bar{Q}$ in the form 31 .

$$
\bar{Q}=f_{c}\left(R_{\lambda}, M_{t}\right)
$$

This has been a basic assumption in substantial amount of research of compressible flows. For example, several theories have been proposed to determine the scaling of so-called dilatational dissipation [15] or the spectrum [4, 7, 32? ] with $R_{\lambda}$ and $M_{t}$. Weaker formulations of the form $\bar{Q}=f_{i}\left(R_{\lambda}\right) f_{c}\left(M_{t}\right)$ are also common. All these different proposals have been tested against numerical simulations with mixed success and no universal behavior has emerged. Part of this state of affairs, we argue, is because of an incorrect identification of relevant nondimensional groups to determine the statistical state of turbulence. While $M_{t}$ compares turbulence velocities to the propagation speed of linear waves, it does not contain any information on, for example, the amount on energy in those compressible modes that may propagate in a wave-like fashion.

\section{THE ROLE OF DILATATIONAL MOTIONS}

The inadequacy of Eq. 2 is evident by the qualitatively distinct behavior observed for turbulence when the nature of the forcing or initial conditions are changed. The structure and dynamics at both terrestrial and astrophysical conditions were found to depend strongly on whether driving forces [3, 16, 33, (check this refs)] or initial state of the flow [8, [9, (check this refs)] contain or not dilatational contributions.

Consider mass conservation

$$
\frac{1}{\rho} \frac{D \rho}{D t}=-\nabla \cdot \boldsymbol{u},
$$

with $D / D t$ being the standard substantial derivative. Using Helmholtz decomposition one can easily see that changes in density following a fluid element can only be 
due to $\boldsymbol{u}_{d}$. Thus, it is clear that one needs a dilatational velocity scale, $\mathcal{U}_{d}$, to properly normalize Eq. 3. Furthermore, in many situations, density and pressure are related through an isentropic or polytropic relation 32 , 34 which would then imply pressure would also be governed by dilatational motions.

In fact, starting with the full Navier-Stokes equations, one can derive evolution equations for the individual components $\boldsymbol{u}_{s}$ and $\boldsymbol{u}_{d}$ 35] where the solenoidal component of velocity and pressure satisfy the incompressible Navier-Stokes equation. The equations for the compressible part of the velocity field is obtained by subtracting the incompressible system from the full set of equations. While these equations are coupled, the time scales and length scales associated with the evolution of each component are expected to be different [23, 36]. In fact for small fluctuations and low Mach numbers, it has been argued that the dilatational component of velocity decouples from its solenoidal component. More formally, and under a slightly different approach, one can linearize the governing equations, assume isentropic fluctuations, and project the velocity field onto the Craya basis in Fourier space 37. In this reference frame, the third axis is defined along the wavenumber vector $\boldsymbol{k}$, and thus the dilatational velocity projects only along that direction. The solenoidal component is divergence free which in Fourier space implies $\hat{\boldsymbol{u}}_{s} \cdot \boldsymbol{k}=0$ (a caret indicates Fourier tranformed variables), and thus lies on the plane perpendicular to it. Then, to leading order, the dilatational velocity and pressure evolve according to:

$$
\begin{aligned}
& \partial u_{d} / \partial t=c_{0} k P_{d} \\
& \partial P_{d} / \partial t=-c_{0} k u_{d}
\end{aligned}
$$

where $u_{d}$ can be written as a scalar since the direction is always along the wavenumber vector. The speed of sound (constant in this simplified case) and the wavenumber are $c_{0}$ and $k$ respectively. $P_{d}=\iota p_{d} / \rho_{0} c_{0}$ is a normalized pressure with $\iota=\sqrt{-1}$. The problem is now simple enough to accept an analytical solution which we will explore momentarily. For now, it suffices to say that, at least to first order, the dilatational motions of the governing equations decouple from solenoidal motions and their dynamics are determined by an interplay between dilatational velocity and pressure. In this approximation, there is no clear reason to believe that a characteristic solenoidal velocity scale would be an appropriate scale for Eq. 4. Similar conclusions can be drawn for the evolution of the different modes based on the Kovasznay decomposition [27.

The accumulated data in the literature, in fact, support these arguments which implies that a relation like Eq. 2 cannot hold in the general case. An example is seen in Fig. 11(a) where we show the variance of pressure normalized by its mean in a statistically steady flow forced stochastically by purely solenoidal forcing (closed circles) and combination of solenoidal and dilatational forcing (closed triangles). Clearly dilatational forcing has a first order effect on the dynamics of the flow generating pressure fluctuations orders of magitude larger than those
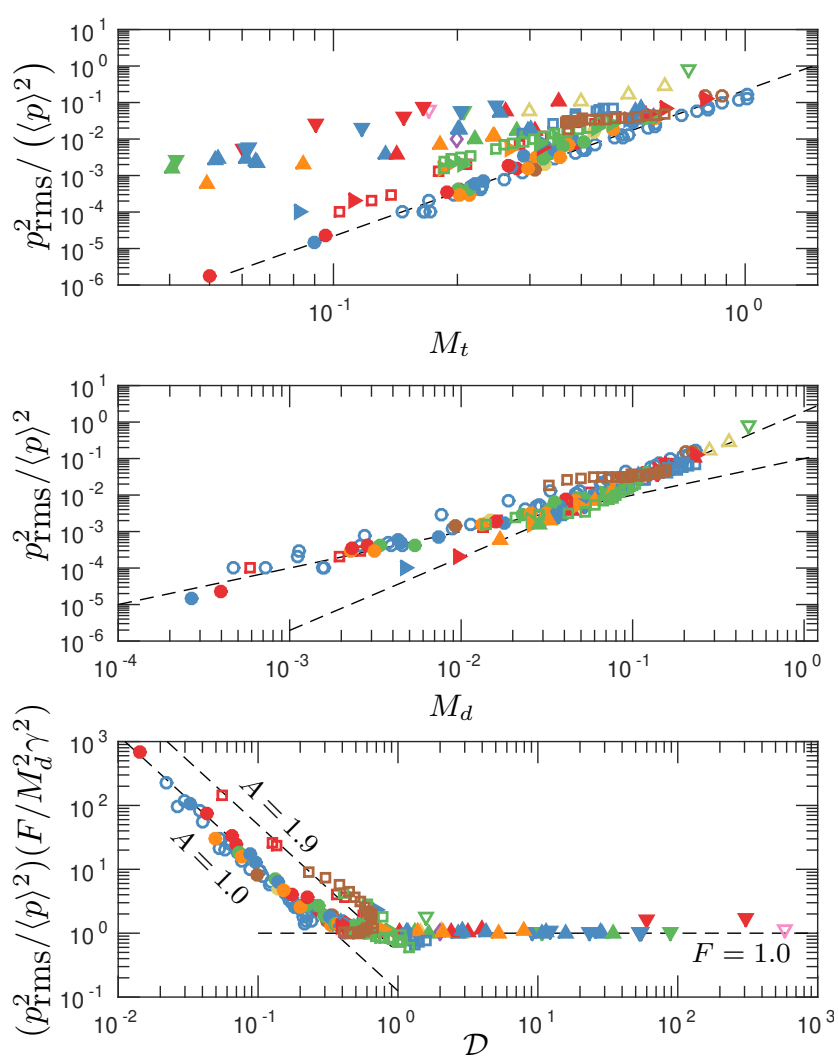

FIG. 1: Scaling of variance of pressure with $M_{t}$ (a), $M_{d}$ (b) and $\mathcal{D}(\mathrm{c})$. In (a), dashed lines are $\gamma^{2} M_{t}^{4} / 9$ [34. In

(b) dashed lines are $\gamma^{2} M_{d}^{2} / F_{T}$ (high $M_{d}$ ) and $c M_{d}$ for reference (low $M_{d}$.) In (c) dashed lines are $\mathcal{D}^{-2}($ low $\mathcal{D}$ )

for different values of $A$ and the asymptotic DDE horizontal lines (high $\mathcal{D})$.

seen at similar $M_{t}$ with solenoidal forcing. Fig. 1 also includes data from a large number of studies which include both solenoidally and dilatationally forced isotropic turbulence as well as shear flows. These are summarized in Table I] It is also clear that the scaling with $M_{t}$ proposed in 34 for solenoidal forcing (dashed line) is inadequate for the general case. As we will see momentarily universal scaling do in fact emerge when one uses an appropriate non-dimensional group which incorporates dilatational motions.

Our data in Fig. 17 come from highly resolved direct numerical simulations (DNS) of the compressible NavierStokes equations representing conservation of mass, momentum and energy. To provide a full set of closed equations we use, as commonly done, a perfect gas to relate thermodynamic quantities, and molecular transport terms with a power-law dependence on temperature. The simulations use tenth-order compact schemes for spatial differentiation and third order Runge-Kutta in time. The momentum equations are forced stochastically at the largest scales (low wavenumbers) using independent Ornstein-Uhlenbeck random processes with finite 


\begin{tabular}{|c|c|c|c|c|}
\hline Flow type & $\sigma$ & $M_{t}$ & $R_{\lambda}$ & Symbol \\
\hline HIT, S [7] & 100 & $0.05-1.02$ & $38-370$ & $\bigcirc$ \\
\hline HIT, S [16] [38] & 100 & $0.11-0.88$ & $12-44$ & $\bigcirc$ \\
\hline HIT, S [39] [40] & 100 & 1.03 & 254 & $\bigcirc$ \\
\hline HIT, S (Present) & 100 & $0.05-0.6$ & $38-430 \dagger$ & $\bullet \bullet \bullet$ \\
\hline HIT, D [16] 38] & 0 & $0.11,0.17$ & $5.4,10$ & $\nabla$ \\
\hline HIT, D [6] & 33.33 & 0.73 & 210 & $\nabla$ \\
\hline HIT, D [4] & 50 & $0.30-0.65$ & $196-234$ & $\Delta$ \\
\hline HIT, D [19] & $4.76,50 \dagger$ & 0.6 & 180 & $\nabla \Delta$ \\
\hline HIT, D (Present) & $70-90$ & $0.04-0.8$ & $30-160 \dagger$ & $\nabla$ \\
\hline HIT, D (Present) & $35-70$ & $0.05-0.55$ & $38-154 \dagger$ & $\Delta \Delta \Delta$ \\
\hline HIT, D (Present) & $0-35$ & $0.04-0.25$ & $16-77 \dagger$ & $\nabla \nabla \nabla$ \\
\hline HST [41] & $\ldots$ & $0.03-0.66$ & $32-220$ & $\square$ \\
\hline HST [42] & $\ldots$ & $0.2-0.5$ & $16-35$ & $\square$ \\
\hline HST [43] & $\ldots$ & $0.2-0.7 \dagger$ & $14-45$ & $\square \square$ \\
\hline HST [17] & $\ldots$ & $0.13-0.65$ & $14-32$ & $\square$ \\
\hline HIT, TF [5] & $\ldots$ & $0.2,0.6$ & 250 & $\diamond$ \\
\hline
\end{tabular}

TABLE I: Databases used in the present study. Flow types: homogeneous isotropic turbulence (HIT), homogeneous shear turbulence (HST, squares), which can have solenoidal forcing (S, circles), some dilatational forcing (D, triangles), or thermal forcing (TF). Studies with multiple symbols correspond to the different conditions marked with a $\dagger$ in increasing order. Studies marked with an asterisk did not provide $\delta$ and was thus computed using pressure fluctuations and Eq. 9.

time correlation. In Fourier space, one can project the Fourier modes on a plane perpendicular to the wavenumber vector or parallel to it, resulting in solenoidal and dilatational forcing, respectively. A statistically stationary state is maintained by removing energy from the system uniformly through the energy equation. We find the results here to be insensitive of whether energy is removed or not or the details of energy removal. Further details of the numerical scheme as well as detailed statistics of the resulting flow can be found in [32, 34].

\section{SELF SIMILARITY FOR COMPRESSIBLE FLOWS}

Returning to the discussion on the general principles around scaling in incompressible turbulence, we are thus confronted with the same difficulties but on a larger parameter space. As argued above, one needs to identify a dilatational velocity $\mathcal{U}_{d}$ that characterize the content of dilatational motions. Unfortunately, it is unclear how this velocity can be obtained from geometrical aspects of a given flow such as the geometry of a grid. And even if it was possible for one particular flow, the results would typically depend on the details of the setup, as we illustrate below. What we propose instead is to rely on the same approach used in incompressible turbulence, namely, to seek internal similarity in which we use an internal scale generated by the flow. A natural choice would be $u_{d, \text { rms }}$, the root-mean-square of the di- latational component of velocity based on the Helmholtz decomposition. This is an analogous reasoning that leads to the selection of $u_{\mathrm{rms}}$ for incompressible flows. The parameter space is now augmented such that

$$
Q=f\left(L, \mu,\langle\rho\rangle, u_{\mathrm{rms}}, c, u_{d, \mathrm{rms}}\right) .
$$

Note that only a single length scale is included here which can be justified in the grounds that the geometry of the device, for example, will provide a natural length scale for the problem with no distinction between solenoidal and dilatational components. The validity of such an assumption will ultimately rest on its success in providing universal scaling laws to numerical and experimental data.

Dimensional analysis would then reduce the list of parameters from six to three. Different non-dimensional groups can be formed containing dilatational motions. Three such parameters are $\delta=u_{d, \mathrm{rms}} / u_{s, \mathrm{rms}}, \quad \chi \equiv$ $K_{d} / K$ (where $K_{d}=\left\langle\rho\left|\boldsymbol{u}_{d}\right|^{2}\right\rangle / 2$ and $K=\left\langle\rho|\boldsymbol{u}|^{2}\right\rangle / 2$ are the turbulent kinetic energy in the dilatational mode and both modes combined, respectively), and $M_{d} \equiv u_{d, \mathrm{rms}} / c$ which compares a typical velocity of dilatational motions to that of acoustic propagation. The parameter $\chi$ is perhaps the most widely used, while $M_{d}$ has been explored in astrophysical contexts 1 . It is trivial to find relationships such as $\chi \approx \delta^{2} /\left(\delta^{2}+1\right)$ or $M_{d} \approx M_{t} \sqrt{\chi}$ where the approximations stem for neglecting density correlations, which have verified to be minor for the objectives of this work. Note that searching for the scaling of these parameters with $M_{t}$, which has been the focus of some investigations, implicitly assumes them to be dependent parameters of the problem. For example, $\chi \sim M_{t}^{2}$ or $M_{t}^{4}$ have been suggested based on different assumptions on EDQNM closures [44. However, as we have seen in Fig. [1 a,$M_{t}$ and $R_{\lambda}$ alone cannot describe completely the statistical state of turbulence in the general case.

Our main objective here is to explore the use of two non-dimensional groups such as $M_{t}$ and $\delta$ as the proper similarity parameters. Formally, we suggest that selfsimilar scaling is possible in compressible flows if internal dilatational scales are included to form similarity parameters. That is, we propose

$$
\bar{Q}=f_{c}\left(R_{\lambda}, M_{t}, \delta\right) .
$$

instead of Eq. 2 as a base of finding universality in such flows. While other non-dimensional parameters can be formed, as discussed above, $M_{t}$ has been used extensively as a governing parameter and is used here complemented by $\delta$.

The addition of $\delta$ as a parameter to characterize compressibility highlights another important aspect that may not be immediately apparent. The turbulent Mach number $M_{t}$ has been traditionally used to characterize two aspects of compressible turbulence. On the one hand, it has been used as a measure of compressibility levels, which can be interpreted as the strength of dilatational motions in the flow. On the other hand, $M_{t}$ has also 
been widely interpreted as the ratio of acoustic to turbulence time scales. This follows from using the so-called eddy-turnover time, $L / u_{\mathrm{rms}}$, to characterize the large turbulent scales and identifying the acoustic time with $L / c$ which represents the time for an acoustic wave to transverse a distance of the order of the largest scales in the flow. The ratio is obviously $M_{t}$ and measures the disparity at which acoustic and turbulence processes occur. A large time disparity (low $M_{t}$ ) was assumed to obtain the linear system in Eq. 4 [35. The dual role traditionally assigned to $M_{t}$, thus, conceptually forbids situations where acoustic phenomena occur at short time scales (low $M_{t}$ ) but strong compressibility effects (high $\delta)$ are present. The alternative use of $M_{d}, \chi$ or $\delta$ to characterize compressibility levels, thus, does not restrict Eq. 4 to flows with small dilatational content.

This may help explain the accuracy and robustness of equipartition ( $F \approx 1$ as shown below) for flows with relatively large values of $M_{t}$ 23, which is unexpected given that equipartition is based on Eq. 4. assumed to be valid only in the low $M_{t}$ limit. However, in 23, as the initial $M_{t}$ was increased, so was $\chi$. It is, thus, possible that at high $\chi$, dilatational terms in the governing equations may dominate the dynamics even though scale separation was not that large (relatively high $M_{t}$ ). Therefore, the linear system Eq. 4 may be valid for a wider range of conditions than originally thought.

\section{THE SCALING OF PRESSURE IN COMPRESSIBLE TURBULENCE}

In Fig. 17 we showed the dramatic effect of dilatational forcing on the variance of pressure. We have also argued, with Eq. 3 and Eq. 4, that fluctuations of thermodynamic variables are more closely related to dilatational motions, and proposed Eq. 6. Here we will look at the specific case of pressure fluctuations for which known statistical equilibrium states can be used to justify more rigorously this proposal.

Consider the pressure variance, which according to Eq. 6 can be written as $p_{\mathrm{rms}}^{2} /\langle p\rangle^{2}=f_{c}\left(R_{\lambda}, M_{t}, \delta\right)$ where the mean pressure is formed with the governing parameters as $\langle p\rangle=c^{2}\langle\rho\rangle / \gamma$. In the incompressible limit it is wellestablished that pressure finds a statistical equilibrium with $p_{\text {rms }} \approx A\langle\rho\rangle u_{s, \text { rms }}^{2}$ with a very weak Reynolds number dependence [45, 46]. Using definitions, this expression can be re-written as

$$
p_{\mathrm{rms}}^{2} /\langle p\rangle^{2} \approx A^{2} \gamma^{2} M_{t}^{4} / 9 .
$$

This scaling has been verified with solenoidally forced simulations in 7, 34 also seen in Fig. 1月 (circles and dashed line).

Now consider the purely dilatational case governed by the system Eq. 4. It is easy to obtain an analytical solution for $u_{d}$ and $p_{d}$. For any initial condition, one can show that for unforced flows the solution tends to an equilibrium state of equipartition between dilatational kinetic energy and potential energy in the dilatational pressure [23]. While in the literature this is simply called equipartition, here we will call it $p$-equipartition to distinguish it from another form of equipartition discussed below. Explicitly, this statistical state can be expressed as $F_{p}=1$ using the so-called equipartition function $F_{p} \equiv c_{0}^{2} \rho_{0}^{2} u_{d, \mathrm{rms}}^{2} / p_{d, \mathrm{rms}}^{2}$. While this result involves only the dilatational pressure, Ref. [32] shows that as compressibility levels increase, the dilatational pressure becomes more dominant. This observation implies that at high compressibility levels (a condition to be defined momentarily), the dilatational pressure would be indeed a proxy to assess the scaling of total pressure. In this case, which we term dilatationally dominated $p$-equipartition, or DDE, we would then expect $p_{\mathrm{rms}}^{2}=c_{0}^{2} \rho_{0}^{2} u_{d, \mathrm{rms}}^{2} / F_{p}$ or in non-dimensional form

$$
p_{\mathrm{rms}}^{2} /\langle p\rangle^{2}=\gamma^{2} M_{d}^{2} / F_{p} .
$$

Clearly this scaling does not conform with Eq. 2 but does agree with the proposed Eq. 6 since $M_{d} \approx M_{t} \delta / \sqrt{\delta^{2}+1}$. In Fig. 1 $1 \mathrm{~b}$ we test the scaling with $M_{d}$. Comparision between parts (a) and (b) reveals a much better collapse of the data on a universal curve at high $M_{d}$. Since the database contains cases where dilatational motions are directly driven by an external dilatational forcing and also cases where dilatational motions appear exclusively due to Navier-Stokes dynamics (solenoidal forcing or homogeneous shear flows), the collapse in the figure supports the idea of universal scaling when an internal dilatational velocity scale is used, regardless of the means of generation. Reynolds number effects are found to be negligible consistent with previous studies [32, 34]

These results have clear implications for the scaling of compressible turbulence. $P$-equipartition, as expressed in Eq. 8, is a theoretical result that follows from neglecting several terms, including those representing viscous and non-linear processes, in the governing equations 23 . The fact that $p$-equipartition is indeed observed at some condition (which is identified below), forces us to conclude that Eq. 2 is fundamentally deficient in the general case.

By construction we have $p=p_{s}+p_{d}$ which implies $p_{\mathrm{rms}}^{2}=p_{s, \mathrm{rms}}^{2}+p_{d, \mathrm{rms}}^{2}+2 r p_{s, \mathrm{rms}} p_{d, \mathrm{rms}}$ where $r$ is the correlation coefficient between $p_{s}$ and $p_{d}$. In the two asymptotic cases discussed above, however, one component of pressure will be much larger than the other: either $p_{\text {rms }} \approx p_{s, \text { rms }}$ or $p_{\text {rms }} \approx p_{d, \text { rms }}$. This will also be the case if the two components of pressure are only weakly correlated as was found at low $M_{t} 32$. We thus have

$$
\frac{p_{\mathrm{rms}}^{2}}{\langle p\rangle^{2}} \approx A^{2} \gamma^{2} \frac{M_{d}^{4}}{\delta^{4}}+\frac{\gamma^{2}}{F} M_{d}^{2}
$$

or, for convenience, one can re-write it in nondimensional form as

$$
\frac{p_{\mathrm{rms}}^{2}}{\langle p\rangle^{2}} \frac{F}{M_{d}^{2} \gamma^{2}} \approx A^{2} F \mathcal{D}^{-2}+1
$$


where the new parameter

$$
\mathcal{D} \equiv \delta \sqrt{\delta^{2}+1} / M_{t}
$$

is a measure of the relative dominance of the dilatational to the solenoidal contributions. We can also write, in terms of different parameters, $\mathcal{D}=\delta^{2} / M_{d}=$ $\chi / M_{d}(1-\chi)=\sqrt{\chi} / M_{t}(1-\chi)$. Note that $\mathcal{D}$ is a complex combination of the originally proposed non-dimensional groups in Eq.6 and can thus be considered a consequence of self-similarity of the second kind [4] in which governing non-dimensional groups cannot be obtained by dimensional analysis alone.

This relation presents some interesting consequences. First, it suggests $\mathcal{D}$ as an appropriate parameter to determine not only levels of pressure fluctuations but also, the statistical regime one expects turbulence to be in. At high $\mathcal{D}$, dilatational pressure dominates and $p$ equipartition is the main mechanism governing the dynamics of pressure fluctuations. At low $\mathcal{D}$, pressure is dominated by its elliptic nature dictated by the incompressible Navier-Stokes equations. In $p$-equipartition one has $F=1$, and since $A \sim O(1)$ for all flows, one would expect a critical value $\mathcal{D}=\mathcal{D}_{\text {crit }}$ of order unity that separates the two regimes. The specific value of $\mathcal{D}_{\text {crit }}$ as well as the asymptote at high $\mathcal{D}$, however, will retain a (weak) dependence on flow characteristics through the constant A.

These observations are indeed consistent with Fig. 17 where we show the data normalized according to Eq. 10. There is excellent collapse of the data along these two asymptotic equilibrium states with a relatively sharp transition around $D_{\text {crit }} \approx 0.5$. This transition towards a flow dominated by dilatational pressure relative to solenoidal pressure was also observed in Ref. [32] (also included here) though the use of $M_{t}$ instead of $\mathcal{D}$ (perhaps justified since forcing was solenoidal and identical in nature for all runs) results in larger scatter.

Fig. 1. also includes homogeneous shear flows from Refs. [17, 41, 43, where no explicit forcing term is added to the governing equations. Instead, turbulent fluctuations are generated as a result of the production mechanism by the mean shear. The good collapse with other data provides further support for universal self-similar scaling for diverse flows driven by different mechanisms.

We do point out, though, that while the constant $A$ in Eq. 7 is order unity, its numerical value is flow dependent. In fact, for the shear flow studies we find $A \approx 1.9$ which is slightly different than 1.2 in stochastically forced isotropic flows 32 . Different low- $\mathcal{D}$ asymptotes are therefore observed for these cases. On the other hand, if full $p$-equipartition governs the flow $(F=1)$, then the asymptotic state is universal.

To put forth Eq. 10 we have assumed that the solenoidal pressure behaves as in incompressible flows and that the dilatational part behaves according to $p$-equipartition for all conditions. This would imply that, for example, $p_{s, \mathrm{rms}}=A\langle\rho\rangle u_{s, \mathrm{rms}}^{2}$ regardless of the amount of dilatation in the flow. However, weaker assumptions are actually needed, namely, that the solenoidal pressure is not affected by the dilatational component when solenoidal pressure dominates the total pressure. Thus, the success of Eq. 10 to collapse the data does not preclude other behavior for $p_{s, \mathrm{rms}}$ when dilatational pressure dominates. The same can be said about dilatational pressure, namely, that it is not affected by the solenoidal part only when dilatational pressure dominates and that other scaling behavior is possible under those conditions.

Another interesting observation from Eq. 11 is the dependence on $M_{t}$. While throughout the literature this is considered to be a measure the level of compressibility, Eq. 11 seems to suggest that, an increase in $M_{t}$ (at constant $\delta$ ) leads to a decrease in $\mathcal{D}$ which would represent weaker compressibility effects. This result and its broader implications will be discussed in the Discussion section.

We finally note that in a number of circumstances loworder statistics of thermodynamic variables can be related to a good approximation by an isentropic, or more generally, polytropic process 4, 7, 32, 34, 41, 48. In this case, one finds $T_{\mathrm{rms}} /\langle T\rangle$ and $\rho_{\mathrm{rms}} /\langle\rho\rangle$ to exhibit the same universal scaling seen in Fig. 1 1 , with slightly different prefactors.

The spatial structure of the flows in the different regimes is also qualitatively different. In Fig. 2 we show the instantaneous pressure gradient at an arbitrary plane and instant of time. The flow is seen to exhibit completely different features even when $M_{t}$ is similar (a and d) showing again the inadequacy of $M_{t}$ to capture important aspects of the flow. In (a) and (b), $\left(\mathcal{D}<\mathcal{D}_{\text {crit }}\right)$ we observe that pressure gradient contours look similar to those seen in incompressible turbulence, consistent with the fact that the pressure field is dominated by the solenoidal pressure. Beyond the transitional $D_{\text {crit }} \approx 0.5$ (panels c and d) we observe very thin high pressure gradient fronts resembling shock waves in contrast to the more isotropic vortical high and low gradient regions observed for cases with $D<D_{\text {crit }}$. However, some differences are also apparent between panels $\mathrm{c}$ and $\mathrm{d}$. The shock-like structures in c appear more curved than those in d indicating perhaps a stronger coupling between the vortical solenoidal motions and the strong compressions present in the flow. As we argue in the Discussion section, this is due to the higher $M_{t}$ in the former.

\section{SMALL-SCALE UNIVERSAL SCALING}

In the classical phenomenology of turbulence, energy is produced at the largest scales (due to the geometry of the device or forcing mechanism). Non-linear mechanisms then lead to instabilities which results in the generation of smaller and smaller scales. This energy transfer process continues until scales are small enough that the smoothing effect of molecular transport processes (viscous effects) become dominant and the energy is dissi- 

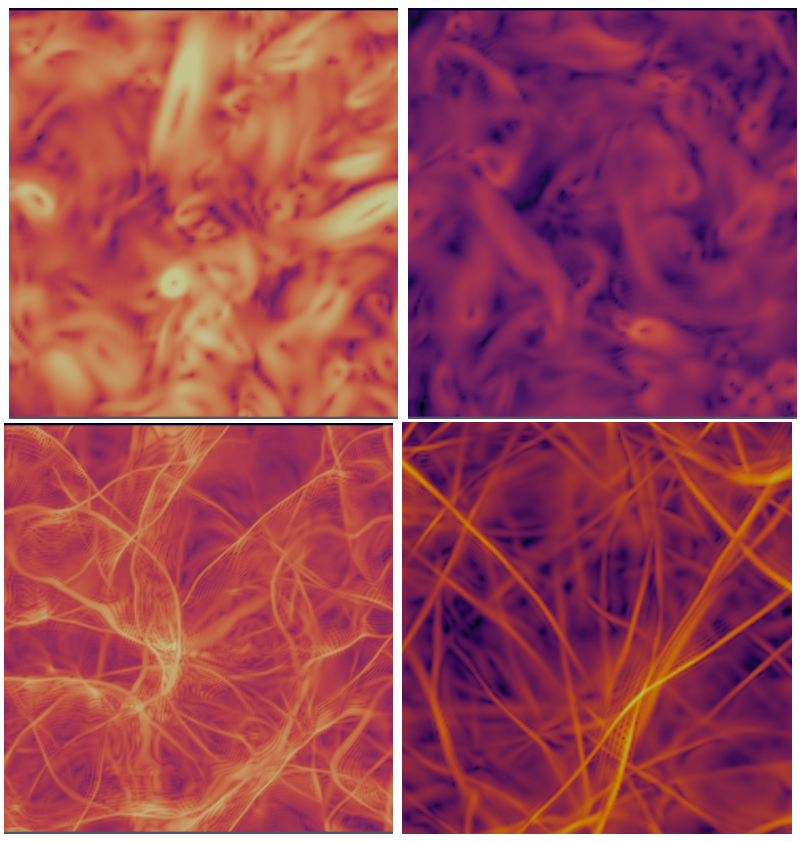

FIG. 2: Contours of $|\nabla p|$ for

$\left(\mathcal{D}, R_{\lambda}, M_{t}, \delta\right)=(0.04,58,0.09,0.0035)(\mathrm{a})$, $(0.27,102,0.36,0.097)(\mathrm{b}),(12,50,0.20,1.4)(\mathrm{c})$, and $(52,51,0.06,1.65)(\mathrm{d})$.

pated into heat. In this step-by-step energy cascade, motions progressively lose information from (non-universal) geometrical aspects at the large scales. Thus, the hope for self-similar universality at small scales.

The result of this energy dissipation at small scales is a temperature increase and a decay of all spatial and temporal fluctuations. The rate at which this happens is dictated by the so-called energy dissipation rate $\langle\epsilon\rangle=2 \mu\left\langle s_{i j} s_{i j}\right\rangle$ (summation convention implied) where $s_{i j} \equiv\left(\partial u_{i} / \partial x_{j}+\partial u_{j} / \partial x_{i}\right) / 2$ is the fluctuating strain rate tensor. Dissipation is also a key ingredient in the classical understanding of turbulent flows as it is the last step in the energy cascade from large scales to small scales [10]. In incompressible homogeneous flows the dissipation can also be written as $\langle\epsilon\rangle=\mu\left\langle\omega_{i} \omega_{i}\right\rangle$ (where $\boldsymbol{\omega} \equiv \nabla \times \boldsymbol{u}$ is the vorticity vector) and its scaling has been the focus of a large body of literature [4] and is relatively well understood. A main result is that $\langle\epsilon\rangle$ becomes independent of viscosity at high Reynolds numbers for the flow configurations considered here. In compressible flows, the situation is more complicated and much less is known [32, 50. due to both the larger parameter space and the additional terms that contribute to dissipation. In particular, if the flow is homogeneous we can write $\langle\epsilon\rangle=\left\langle\epsilon_{s}\right\rangle+\left\langle\epsilon_{d}\right\rangle$ where $\left\langle\epsilon_{s}\right\rangle \equiv\left\langle\mu \omega_{i} \omega_{i}\right\rangle$ and $\left\langle\epsilon_{d}\right\rangle \equiv(4 / 3)\left\langle\mu \theta^{2}\right\rangle$ with $\theta \equiv \partial u_{i} / \partial x_{i}$ being the dilatation. The first component is the so-called solenoidal dissipation as its expression is identical to that in incompressible flows. Clearly, only the solenoidal component of the velocity contributes to $\left\langle\epsilon_{s}\right\rangle$ as the dilatational component is irrotational by construction. The second component is the so-called dilatational dissipation and is exclusively due to the dilatational velocity. The historical focus has largely been in understanding and modeling the dilatational dissipation as a "correction" due to compressibility [15. The most widely used models are of the form $\left\langle\epsilon_{d}\right\rangle \propto\left\langle\epsilon_{s}\right\rangle M_{t}^{\alpha} R_{\lambda}^{\beta}$ where different models lead to different exponents (e.g. $(\alpha, \beta)=(2,0)$ [23], $(4,-2)$ [36], or $(5,0)$ [7]) though other more general functional forms of the type $\left\langle\epsilon_{d}\right\rangle=\left\langle\epsilon_{s}\right\rangle F\left(M_{t}\right)$ have been also proposed [15, 44. However, when the available data are collected together as in Fig. $3 \mathrm{a}$, it is apparent that any model following the similarity scaling in Eq. 2 will be unable to capture a universal behavior.

Phenomenologically, since dissipation is proportional to velocity gradients, one can estimate the scaling of each component, in view of Eq. 5, as $\left\langle\epsilon_{s}\right\rangle \sim \mu\left(u_{s, \mathrm{rms}} / L\right)^{2} g_{1}\left(R_{\lambda}, M_{t}, \delta\right)$ and $\left\langle\epsilon_{d}\right\rangle \sim$ $\mu\left(u_{d, \mathrm{rms}} / L\right)^{2} g_{2}\left(R_{\lambda}, M_{t}, \delta\right)$ where $g_{1}$ and $g_{2}$ are some presumably universal scaling functions. The ratio is then $\left\langle\epsilon_{d}\right\rangle /\left\langle\epsilon_{s}\right\rangle=\delta^{2} g_{3}\left(R_{\lambda}, M_{t}, \delta\right)$ where $g_{3}$ is another universal function. In standard models, then, we have $g_{3} \propto R_{\lambda}^{\alpha} M_{t}^{\beta} \delta^{-2}$. To assess the scaling with the governing parameters in Fig. $3 \mathrm{~b}$ we show the ratio of dissipation rates versus $\delta$. We see that the all data from different flows, with different forcing schemes, and different conditions seem to collapse along

$$
\left\langle\epsilon_{d}\right\rangle /\left\langle\epsilon_{s}\right\rangle \approx \delta^{2},
$$

(i.e. $g_{3} \approx 1.0$ ). This scaling seems robust for almost 10 order of magnitude in $\left\langle\epsilon_{d}\right\rangle /\left\langle\epsilon_{s}\right\rangle$ for all flows (including shear flows). Whil some scatter is observed in the data, no distinguishable systematic trends with the other parameters can be seen. This again highlights the inadequacy of Eq. 22 and adequacy of Eq. 6, and provides strong support for broad universality when dilatational motions are included in the set of governing parameters. For later use, we note that dilatational dissipation becomes larger than solenoidal dissipation at $\delta \approx 0.9$.

We finally turn to the skewness of longitudinal velocity gradients $S=\left\langle\left(\partial u_{1} / \partial x_{1}\right)^{3}\right\rangle /\left\langle\left(\partial u_{1} / \partial x_{1}\right)^{2}\right\rangle^{3 / 2}$ which has also been studied extensively in incompressible flows [26]. The fact that $S$ is non-zero is a manifestation of the non-Gaussianity of the velocity field. Physically, it represent a normalized measure of the production of rotational motions or enstrophy $\left(=\left\langle\omega^{2}\right\rangle\right)$ due to non-linear mechanisms. Its value is found to be an approximately universal constant around -0.5 for a range of incompressible flows at different conditions [26. In fact, it is so robust a measure, that it is common to assess the emergence of "realistic" turbulence by examining the value of $S$ in simulations and experiments. This practice has also been extended to compressible turbulence, though, its interpretation is more involved since, at present, it is unknown how $S$ scale with different parameters. Thus, values not consistent with the incompressible $S \approx-0.5$ could be interpreted as a compressibility effect [7, 40, 51, as an indication that fully developed turbulence has not been achieved [52, or even as a numerical artifact [40]. 

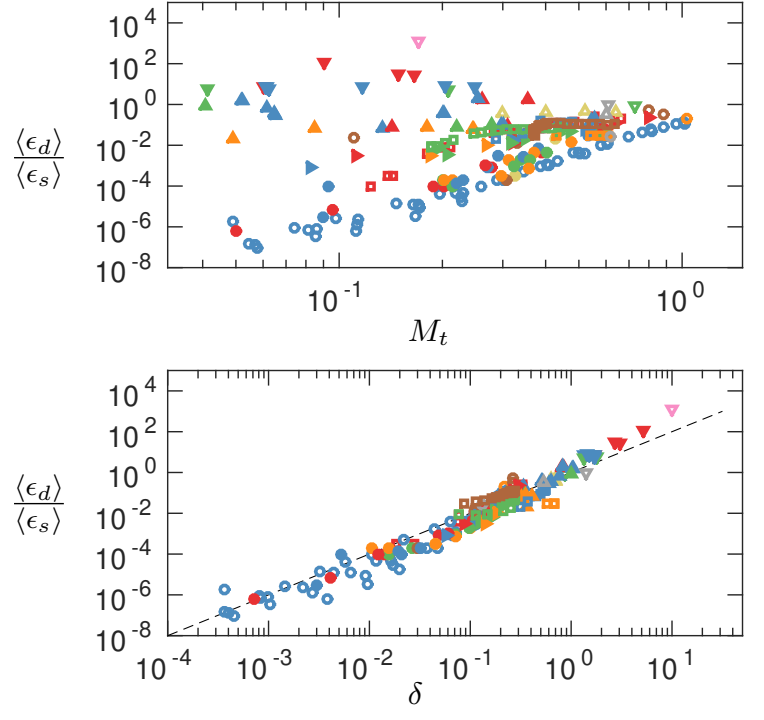

FIG. 3: Scaling of ratio of dilatational to solenoidal dissipation with (a) $M_{t}$ and (b) $\delta$. Dashed line in (b) with a slope of 2 for reference.

Thus, the search for universal scaling in compressible turbulence, has also important practical implications.

In Fig. 4 a, we show the collection of $S$ as a function of $M_{t}$. The familiar lack of universality observed before emerges here too. Under solenoidal forcing and low $M_{t}, S$ is consistent with its incompressible value which implies that under these conditions dilatational motions are either very weak or have equal propensity to form both compressions and expansions. A number of authors [7, 40, 51] have reported larger values of $S$ at higher $M_{t}$ under solenoidal forcing which have been attributed to shocklets or small scale compressions [53. We see here that under dilatational forcing, large negative skewness is observed even at low $M_{t}$ which highlights, as before, the important role of dilatational motions and the need to extend the parameter space. Consistent with this observation is the work of 54 where it has also been observed that dilatational excitation leads to larger fractal dimensions (corresponding to structures between filaments and sheets) as opposed to the more narrow filaments observed with solenoidal forcing.

If the increased dilatational motions are responsible, through shocklets, of the larger value of the gradient skewness then we would expect wave-steepening mechanisms to be significant. This mechanism is represented by the non-linear term $\boldsymbol{u}_{d} \cdot \nabla \boldsymbol{u}_{d}$. To estimate the order of magnitude of this terms, we first note that $\boldsymbol{u}_{d}$ is of order $u_{d, \mathrm{rms}} \sim \delta \boldsymbol{u}_{s}$. The multiple scales expansion of [55], in which the solenoidal and dilatational components act at different scales, leads to a split gradient operator $\nabla=\nabla_{\eta}+M_{t} \nabla_{\xi}$ where $\eta$ and $\xi$ are short and long wavelength scales, being the latter the one corresponding to
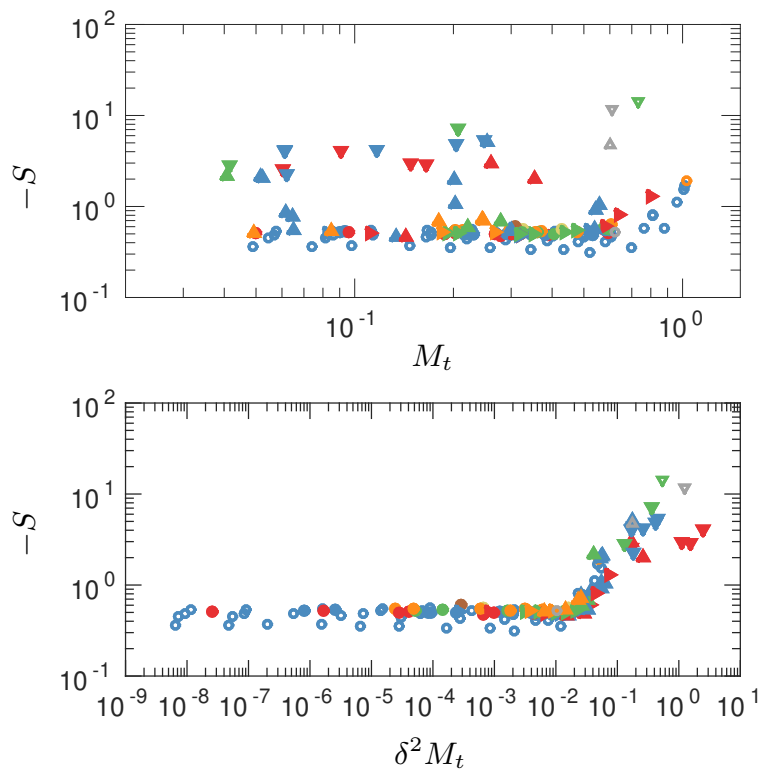

FIG. 4: Skewness of longitudinal velocity gradient

the acoustic contributions. If we consider only that component we can estimate the order of magnitude of the non-linear term as $\delta^{2} M_{t}$. This phenomenological argument would imply that when $\delta^{2} M_{t}$ is high enough, $S$ will increase in magnitude. This is indeed what we see in Fig. $4 \mathrm{~b}$ where, at low values of this parameter, data agree with the incompressible value but diverges at a critical value of $\delta^{2} M_{t} \approx 3 \times 10^{-2}$. This $S$-divergence at a critical value of $\delta^{2} M_{t}$, though inspired in a somewhat crude order-ofmagnitude estimate, is consistent with all other available data in the literature.

\section{DISCUSSION: THE BROADER PICTURE}

In previous sections we showed that the statistical state of compressible turbulence cannot be described by the Reynolds and turbulent Mach numbers alone. Instead, a characteristic dilatational velocity needs to be incorporated into the governing parameters to find universal scaling laws. This approach was shown to collapse data for fluctuations of thermodynamic variables, dissipation rates, and skewness of velocity gradients. In doing so, we identified transitions between equilibrium states which can be used to distinguish different regimes. These regimes are shown in the inset of Fig. 5(a) with lines separating dilatationally-dominated $p$-equipartition (DDE), the divergence of skewness, and the dominance of dilatational dissipation. The main part of the figure contains all the data from Table I in the $\delta$ - $M_{t}$ plane.

This figure clearly shows how $M_{t}$ is unable to determine the state of turbulence in general. Unlike classical problems in critical phenomena, no single transition 
from "incompressible" to "compressible" can be identified. For example, a flow at low $M_{t}$ will, as $\delta$ increases, first transition to DDE, then it will experience $S$-divergence and only at higher levels of $\delta$, dilatational dissipation will start dominating the conversion of kinetic energy to internal energy. At higher $M_{t}$, on the other hand, $S$-divergence may occur even before DDE. We see examples of both regimes in Fig. 5(a): the high$M_{t}\left(M_{t} \gtrsim 0.8\right)$ cases from [7] have much higher values of $S$ than incompressible flows though pressure is not dominated by dilatational dynamics while our low- $M_{t}$ $\left(M_{t} \lesssim 0.4\right)$ dilatationally forced isotropic cases as well as some intermediate- $M_{t}$ shear cases [41, are in DDE but $S$ has not diverged yet. Clearly, comparisons between these flows could lead to misleading conclusions especially if they are at nominally the same $M_{t}$.

Note that the $S$-divergence occurs, except for exceptional conditions (extremely low $M_{t}$ and high $\delta$ ), before dissipation is dominated by dilatational motions. If shocklets are responsible for both, as commonly argued, then their contribution to the third order moment of velocity gradients emerges earlier than to the second moment. This observation seems to be consistent with recent work [56] suggesting that small-scale high- $R_{\lambda}$ features characterized by the anomalous scaling of highorder statistical moments in incompressible flows emerge at lower $R_{\lambda}$ than low-order moments. In subsequent work [57, this transition from two statistical steady states (Gaussian at low $R_{\lambda}$ and anomalous at high $R_{\lambda}$ ) provided the key ingredients to obtain the numerical value of the scaling exponents in the anomalous regime. In this context, the discovery of a transition such as that for $S$ (Fig. 4) is important as it identifies appropriate scaling parameters as well as a seemingly universal transition point. This could, following [57, provide a fruitful venue to completely characterize small-scale behavior in compressible flows.

The collection of all the data in Fig. 5(a) spans virtually all possible regimes. However, individually, a given flow appears to transverse the $\delta-M_{t}$ space along a specific trajectory. For illustration purposes we isolate a subset of the database in Fig. 5(b) comprising different flow geometries and type of forcing: the present isotropic simulations with stochastic solenoidal and dilatational forcing, the isotropic flows of [7] with a low-wavenumber deterministic forcing, the homogeneous shear flow of [4] and [43, and isotropic simulations with forcing that keeps the ratio of dilatation and solenoidal energy constant at low wavenumbers [4.

From Fig. 5(b) we clearly see that different driving mechanisms or geometries generate different levels of dilatations. And for a given flow, this depends on the specific conditions specified by the governing parameters. For example, as the Reynolds and Mach numbers are varied (at constant shear rate) in the HST flows of [41] (red squares), we see an increase in both $M_{t}$ and $\delta$ following an approximate power law of the form $M_{t} \sim \delta^{1 / 3}$ (line II) or, equivalently, $\delta \sim M_{t}^{3}$ at low $\delta$ but appears to transition
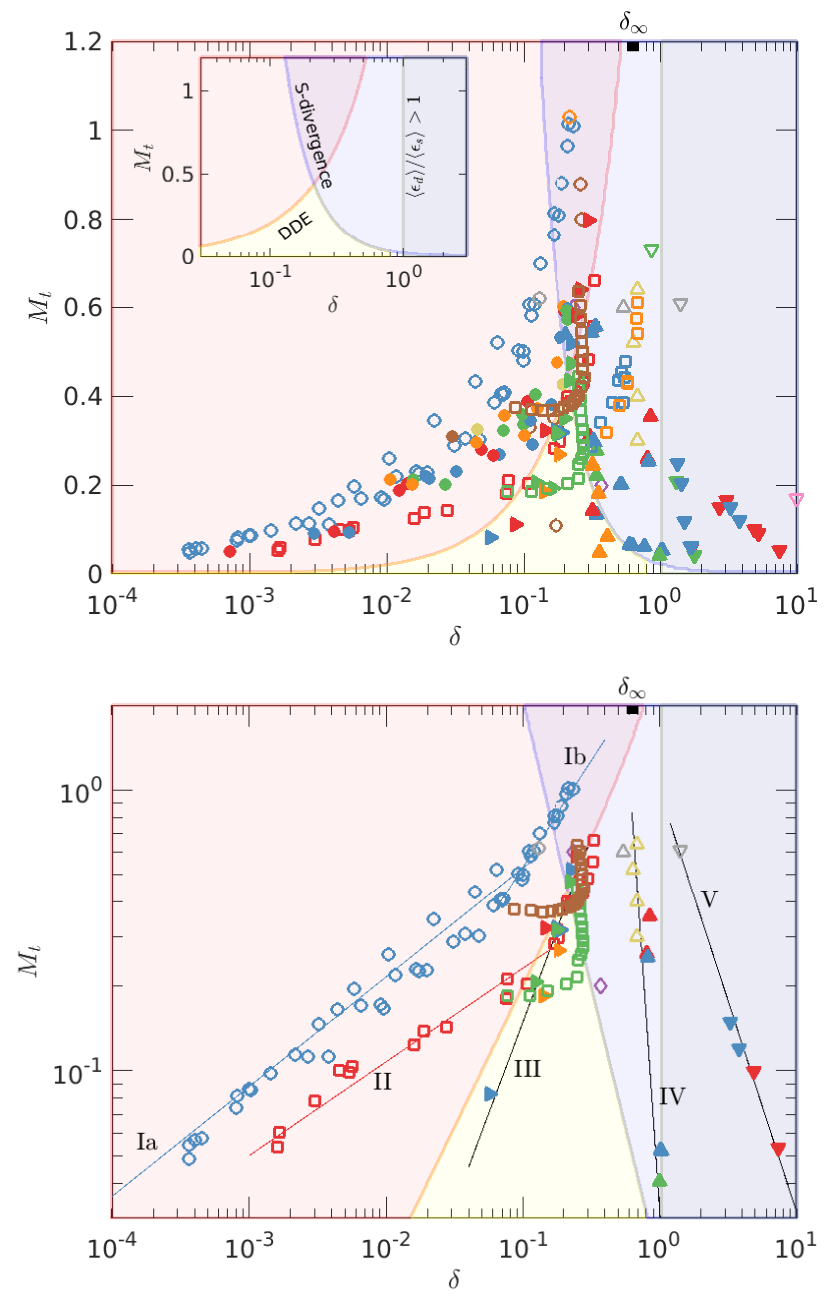

FIG. 5: Regions in the $\delta-M_{t}$ diagram: Dilatationally dominated $p$-equipartition $\left(\mathrm{DDE}, \mathcal{D}>\mathcal{D}_{\text {crit }}\right)$,

$S$-divergence $\left(\delta^{2} M_{t}>2 \times 10^{-2}\right)$, and $\left\langle\epsilon_{d}\right\rangle /\left\langle\epsilon_{s}\right\rangle>1$. (a)

Entire database in TableI. (b) Selected trajectories

which include isotropic cases with two types of

solenoidal forcing, cases with dilatational forcing, and homogeneous shear flows.

to the DDE line at $\mathcal{D}=0.5$ (i.e. $M_{t}=0.5 \delta \sqrt{\delta^{2}+1}$ ) as compressibility levels increase, or to a constant $\delta$ (more below). This behavior is different from the isotropic data of [7] (blue circles) which follow $\delta \sim M_{t}^{2.56}$ at low $\delta$ (line Ia) and transitions to a shallower exponent $\delta \sim M_{t}^{1.33}$ (line Ib) at higher compressibility levels never reaching DDE. Compounded with this, the differences in the prefactors in the expressions relating $\delta$ and $M_{t}$ result in orderof-magnitude differences in the value of $\delta$ for the same $M_{t}$. The trajectory is also different for the isotropic simulation of [4] (yellow triangles) which employ a forcing mechanism that leads to constant $\delta$ as $M_{t}$ is varied. We also include two cases of the temporally evolving shear layers of 43] (green and brown squares) starting from different $M_{t}$, respectively. In each case the flow evolves 
increasing $\delta$ for $M_{t}$ relatively constant at early times but approaches an approximately vertical line (constant $\delta$ ) as $M_{t}$ keeps increasing. Finally we see that the dilatationally forced cases presented here (right, up and down triangles for $\sigma=10 \%, 40 \%$ and $90 \%$ dilatational forcing, respectively) present different qualitative behavior. At low dilatational forcing $(\sigma=10 \%), \delta$ increases as $M_{t}$ increases (line III) consistent with the other flows, though with a smaller exponent $\left(\delta \sim M_{t}^{0.77}\right)$. However, for strongly dilatationally forced flows, the trend is the opposite with $\delta$ decreasing with $M_{t}$ as $\delta \sim M_{t}^{-0.67}$ (line $\mathrm{V})$. At intermediate dilatational forcing $(\sigma=40 \%$, line IV), $\delta$ is approximately constant and close to the data of [4] which was driven by $50 \%$ dilatational forcing.

Obviously understanding the detailed physical mechanisms that lead to specific trajectories in the $\delta-M_{t}$ plane is interesting in its own and their investigation will certainly lead to deeper understanding of the dynamics of particular flow configurations. Our purpose here, however, is precisely to show that $M_{t}$ is insufficient to characterize completely the statistical state of turbulence but that in combination with $\delta$ we can obtain, for example, universal scaling laws for the pressure variance, energy dissipation, and skewness of velocity gradients regardless of the generation mechanism.

This general view of universal statistical equilibria for compressible turbulence in the $\delta$ - $M_{t}$ plane can also be used to help contextualize results in the literature. We have pointed out that different flows follow different trajectories. However, it is also possible to distinguish trajectories (or some of its features) that share commonalities for a given class of systems. For example, solenoidally forced isotropic turbulence may follow $\delta=C M_{t}^{\alpha}$ (at least for some region in the $\delta$ - $M_{t}$ plane) with the same exponent $\alpha$ but different prefactors $C$ depending on the specific form of the driving mechanism. The addition of dilatational forcing changes the numerical value (and even sign) of the scaling exponents $\alpha$, as discussed above. In support of the concept of classes, we note that data from diverse studies in the literature (see symbols in Table I) using different forcing and numerical schemes seem to move along the same trajectories (e.g. lines III, IV and V). Similar considerations could be applied to shear layers. An implication of this is that, within a class, Eq. 6reduces to Eq. 2 and proposals in the literature based on $M_{t}$ may still be approximately valid. The investigation of these trajectories and identification of universal classes would require more data that currently available from simulations and experiments carefully designed for this purpose. This is obviously an important task that would be extremely valuable in turbulence modeling. The data in Fig. 5 while limited is, in a very broad sense, consistent with the existence of classes.

In our search for universal features for compressible turbulence, we finally consider two theoretical results. First, Staroselsky et. al [58] studied isotropic compressible turbulence with a Gaussian forcing at the large scales using renormalization group. A statistical equilibrium was found with a constant ratio of solenoidal and dilatational kinetic energy (that is, constant $\delta$ ) when the forcing spectrum decays sufficiently fast with wavenumber. In particular, they predict an asymptotic limit $\left(\delta \rightarrow \delta_{\infty}\right)$ given by $\delta_{\infty}^{-2} \approx 3$ or $\delta_{\infty} \approx 0.58$. This state of equipartition, which is of different nature than $p$-equipartition, will be called $\delta$-equipartition. The second earlier study is that of Kraichnan [59] who also suggested $\delta$-equipartition but based on statistical mechanics principles. In particular, based on Liouville theorem, he suggested that in the inviscid case and with weak excitation there is equipartition between vortical solenoidal modes (with two degrees of freedom) and acoustic modes, also with two degrees of freedom (one from the dilatational mode and one from a bijective function of the density mode). Thus, $\delta_{\infty}^{2}=1 / 2$ or $\delta_{\infty}=0.7$, which is not far from [58], though derived from a completely different perspective. It is interesting that in the case of weak fluctuations of thermodynamic variables, the more general formulation of Kraichnan also leads to a form of $p$-equipartition.

The range formed by these two theoretical limits is marked on the top of both panels of Fig. 5 with a thick line. Collectively, the data seem qualitatively consistent with such a limit as a universal feature across flows. For example, we see this trend in naturally forced flows such as the shear layers of 43 where $\delta$ clearly approaches a constant not far from the theoretical prediction (apparently independent of initial $M_{t}$ ) as $M_{t}$ increases during the temporal evolution of the flow. It is also interesting that in our isotropic simulations, when dilatational forcing is strong and kept constant, and temperature and viscosity are varied to achieve higher $M_{t}, \delta$ decreases (line IV in Fig. 5b). Even with purely dilatational forcing, the values of $\delta$ seem bounded as $M_{t}$ increases. Further support for such a universal asymptotic behavior is provided by the very-high- $M_{t}$ simulations of [1] that, while different from all in Table $\mathrm{I}$ since they are based on the Euler equations and the flow is isothermal, yield $\delta \approx 0.5$, very close to the the theoretical predictions, as $M_{t}$ is increased to values as high as 15 .

These observations can shed light on the proper interpretation of the governing parameters. In the traditional interpretation, $M_{t}$ is a measure of both, separation of time scales and compressibility level. As we argued above this is inadequate. Instead, $\delta$ is the appropriate non-dimensional group that represents compressiblity levels (dilatational motions), while $M_{t}$ represents the ratio of time scales. When a vast disparity of time scales is present, one would expect little interaction between solenoidal and dilatational phenomena. In fact, when the equations of motion are decomposed into solenoidal and dilatational modes, the expansion of [55] suggests that the cross-terms responsible for the energy exchange between the two modes grow with $M_{t}$. Thus, a simple physical picture emerges based on a universal $\delta$-equipartition state: if flow conditions are such that $\delta \neq \delta_{\infty}$ then an increase of $M_{t}$ will lead to stronger non-linear interactions between solenoidal and dilatational modes that would en- 
able stronger redistribution of energy towards a complete $\delta$-equipartition [59]. If $\delta<\delta_{\infty}$, the stronger interaction will lead to transfers from the solenoidal to the dilatational mode. If $\delta>\delta_{\infty}$ the transfer would proceed in the opposite direction. This phenomenological argument is consistent with the data in Fig. 5 and also with the observations made about the stronger interaction of vortical motions with compression waves giving rise to the more curved spatial structures in Fig. 2 at higher $M_{t}$ but the same $\delta$. Rigorous studies of energy transfers from the governing equations are certainly warranted.

It is also interesting, and perhaps counterintuitive, to see that at high $M_{t}\left(M_{t}>\delta_{\infty} \sqrt{\delta_{\infty}^{2}+1} / \mathcal{D}_{\text {crit }} \approx 1.34\right)$, $\delta_{\infty}$ is to the left of the DDE line. Thus, if $\delta$-equipartition is indeed a universal asymptote at high $M_{t}$, then the solenoidal component will always dominate pressure, regardless of how high $M_{t}$ is. In fact, the higher the $M_{t}$, the more dominant solenoidal pressure becomes $(\mathcal{D}$ decreases) as one transverse the $\delta-M_{t}$ plane with $\delta=\delta_{\infty}$.

In conclusion, using a large database of new direct numerical simulations of isotropic compressible turbulence with different driving mechanisms combined with an extensive set of flows in the literature (including shear flows), we show that universal scaling laws can indeed be identified for compressible turbulence if dilata- tional motions are incorporated in the non-dimensional groups used to determine its statistical equilibrium state. Whereas the traditional turbulent Mach number fails to describe the state of the turbulence and thus to collapse the data for different conditions and flows, we proposed a $\delta$ - $M_{t}$ plane in which different statistical equilibria can be identified, regardless of the flow configuration and geometrical details. Different flows transverse this plane in different ways, but one can postulate classes of systems which may share universal trajectories or scaling exponents defining those trajectories. An ultimate asymptotic regime predicted by renormalization group and statistical mechanics is not inconsistent with available data. The successful collapse of all the available the data (pressure variance, energy dissipation, enstrophy generation), and the identification of the proper parameters to encounter universal scaling laws, open the door to both accurate models and deeper understanding of compressible turbulence.

\section{ACKNOWLEDGMENTS}

This work was partially supported by the National Science Fundation Grant xxxxx and by the Air Force Office of Scientific Research Grant xxxx.
[1] L. Konstandin, P. Girichidis, C. Federrath, and R. S. Klessen, The Astrophysical Journal 761, 149 (2012).

[2] C. Federrath, R. S. Klessen, and W. Schmidt, Astrophys. J. Lett. 688, L79 (2008).

[3] C. Federrath, R. S. Klessen, and W. Schmidt, Astroph. J. 692, 364 (2009).

[4] J. Wang, M. Wan, S. Chen, C. Xie, and S. Chen, Phys. Rev. E 97, 043108 (2018)

[5] J. Wang, M. Wan, S. Chen, C. Xie, L.-P. Wang, and S. Chen, Journal of Fluid Mechanics 867, 195215 (2019)

[6] J. Wang, Y. Yang, Y. Shi, Z. Xiao, X. T. He, and S. Chen, Journal of Turbulence 14, 21 (2013).

[7] J. Wang, T. Gotoh, and T. Watanabe, Phys. Rev. Fluids 2, 013403 (2017)

[8] S. Pirozzoli and F. Grasso, Phys. Fluids 16, 4386 (2004).

[9] R. Samtaney, D. I. Pullin, and B. Kosovic, Phys. Fluids 13, 1415 (2001).

[10] U. Frisch, Turbulence (Cambridge University Press, 1995).

[11] K. R. Sreenivasan, Proc. Nat. Acad. Sci. , 201800463 (2018).

[12] P. Moin and K. Mahesh, Annu. Rev. Fluid Mech. 30, 539 (1998).

[13] T. Ishihara, T. Gotoh, and Y. Kaneda, Annu. Rev. Fluid Mech. 41, 165 (2009).

[14] S. K. Lele, Annu. Rev. Fluid Mech. 26, 211 (1994).

[15] T. B. Gatski and J.-P. Bonnet, Compressibility, turbulence and high speed flow, 2nd ed. (Elsevier, 2013).

[16] S. Kida and S. A. Orszag, J. Sci. Comp. 5, 85 (1990).

[17] S. Sarkar, Journal of Fluid Mechanics 282, 163186 (1995)
[18] A. W. Vreman, N. D. Sandham, and K. H. Luo, Journal of Fluid Mechanics 320, 235258 (1996).

[19] Q. Ni, Physical Review E 93, 043116 (2016).

[20] D. S. Praturi and S. S. Girimaji, Physics of Fluids 31, 055114 (2019).

[21] G. A. Blaisdell, N. N. Mansour, and W. C. Reynolds, J. Fluid Mech. 256, 443 (1993).

[22] J. R. Ristorcelli and G. A. Blaisdell, Phys. Fluids 9, 4 (1997).

[23] S. Sarkar, G. Erlebacher, M. Y. Hussaini, and H. O. Kreiss, J. Fluid Mech. 227, 473 (1991).

[24] A. N. Kolmogorov, Dokl. Akad. Nauk. SSSR 30, 299 (1941).

[25] A. S. Monin and A. M. Yaglom, Statistical Fluid Mechanics, Vol. II (MIT Press, 1975).

[26] K. R. Sreenivasan and R. A. Antonia, Annu. Rev. Fluid Mech. 29, 435 (1997).

[27] A. J. Smits and J. P. Dussauge, Turbulent shear layers in supersonic flow (Springer, 2006).

[28] J. Hall, P. Dimotakis, and H. Rosemann, AIAA journal 31, 2247 (1993).

[29] P. Dimotakis, High Speed Flight Propulsion Systems 137, 265 (1991).

[30] J. B. Freund, S. K. Lele, and P. Moin, Journal of Fluid Mechanics 421, 229 (2000).

[31] A more general list of parameters would include the ratio of specific heats, $\gamma$ but is not included here for simplicity.

[32] S. Jagannathan and D. A. Donzis, J. Fluid Mech. 789, 669 (2016)

[33] W. Schmidt, C. Federrath, M. Hupp, S. Kern, and J. C. Niemeyer, A\&A 494, 127 (2009) 
[34] D. A. Donzis and S. Jagannathan, J. Fluid Mech. 733, 221 (2013)

[35] G. Erlebacher, M. Y. Hussaini, H. O. Kreiss, and S. Sarkar, Theoret. Comput. Fluid Dynamics 2, 73 (1990).

[36] J. R. Ristorcelli, J. Fluid Mech. 347, 37 (1997).

[37] A. Simone, G. N. Coleman, and C. Cambon, J. Fluid Mech. 330, 307 (1997)

[38] S. Kida and S. A. Orszag, J. Sci. Comp. 7, 1 (1992).

[39] J. Wang, Y. Shi, L.-P. Wang, Z. Xiao, X. T. He, and S. Chen, Phys. Rev. Lett. 108, 214505 (2012).

[40] J. Wang, Y. Shi, L.-P. Wang, Z. Xiao, X. He, and S. Chen, Phys. Fluids 23, 125103 (2011).

[41] S. Chen, J. Wang, H. Li, M. Wan, and S. Chen, Physics of Fluids 30, 065109 (2018).

[42] S. Sarkar, G. Erlebacher, and M. Y. Hussaini, Theoretical and Computational Fluid Dynamics 2, 291 (1991).

[43] S. Sarkar, G. Erlebacher, and M. Hussaini, "Compressible homogeneous shear: simulation and modeling. in turbulent shear flows 8 (ed. f.durst et al.)." in Turbulent Shear Flows 8 (Springer, 1993) pp. 249-267.

[44] P. Sagaut and C. Cambon, Homogeneous Turbulence Dynamics (Cambridge University Press, Cambridge, 2008).

[45] L. D. Landau and E. M. Lifshitz, Fluid Mechanics (Pergamon Press, 1987).

[46] D. A. Donzis, K. R. Sreenivasan, and P. K. Yeung, Phys- ica D 241, 164 (2012).

[47] G. I. Barenblatt and Y. B. Zeldovich, Annu. Rev. Fluid Mech. 4, 285 (1972).

[48] J. Wang, T. Gotoh, and T. Watanabe, Phys. Rev. Fluids 2, 053401 (2017)

[49] J. C. Vassilicos, Annual Review of Fluid Mechanics 47, 95 (2015).

[50] G. L. Eyink and T. D. Drivas, Phys. Rev. X 8, 011022 (2018)

[51] J. Wang, T. Gotoh, and T. Watanabe, Phys. Rev. Fluids 2, 023401 (2017).

[52] S. Lee, S. K. Lele, and P. Moin, Phys. Fluids 4, 1521 (1992).

[53] S. Lee, S. K. Lele, and P. Moin, Phys. Fluids 3, 657 (1991).

[54] L. Konstandin, C. Federrath, R. S. Klessen, and W. Schmidt, J. Fluid Mech. 692, 183 (2012).

[55] G. P. Zank and W. H. Matthaeus, Phys. Fluids 3, 69 (1991).

[56] V. Yakhot and D. A. Donzis, Phys. Rev. Lett. 119, 044501 (2017)

[57] V. Yakhot and D. A. Donzis, Physica D. 384-385, 12 (2018)

[58] I. Staroselsky, V. Yakhot, S. Kida, and S. A. Orszag, Phys. Rev. Lett. 65, 171 (1990).

[59] R. H. Kraichnan, J. Acous. Soc. Amer. 27, 438 (1955) 